\title{
Gain characteristics of a saturated fiber optic parametric amplifier
}

\author{
Rottwitt, Karsten; Lorenzen, Michael Rodas; Noordegraaf, Danny; Peucheret, Christophe
}

Published in:

Anniversary International Conference on Transparent Optical Networks

Link to article, DOI:

10.1109/ICTON.2008.4598371

Publication date:

2008

Document Version

Publisher's PDF, also known as Version of record

Link back to DTU Orbit

Citation (APA):

Rottwitt, K., Lorenzen, M. R., Noordegraaf, D., \& Peucheret, C. (2008). Gain characteristics of a saturated fiber optic parametric amplifier. In Anniversary International Conference on Transparent Optical Networks (pp. 62-64). IEEE. https://doi.org/10.1109/ICTON.2008.4598371

\section{General rights}

Copyright and moral rights for the publications made accessible in the public portal are retained by the authors and/or other copyright owners and it is a condition of accessing publications that users recognise and abide by the legal requirements associated with these rights.

- Users may download and print one copy of any publication from the public portal for the purpose of private study or research.

- You may not further distribute the material or use it for any profit-making activity or commercial gain

- You may freely distribute the URL identifying the publication in the public portal 


\title{
Gain Characteristics of a Saturated Fiber Optic Parametric Amplifier
}

\author{
Karsten Rottwitt, Michal Lorenzen, Danny Noordegraff, Christophe Peucheret \\ DTU Fotonik, Department of Photonics Engineering, Technical University of Denmark \\ DK-2800 Lyngby, Denmark, kar@com.dtu.dk
}

\begin{abstract}
In this work we discuss saturation performance of a fiber optic parametric amplifier. A simple numerical model is described and applied to specific cases. A system experiment using a saturated amplifier illustrates a $4 \mathrm{~dB}$ improvement in required signal to noise ratio for a fixed bit error ratio.
\end{abstract}

\section{INTRODUCTION}

Fiber optical parametric amplifiers are promising candidates as key enablers for many all optical fiber subsystems in the future, not only may these provide gain, but they may also perform various other functionalities at the same time, such as wavelength conversion, optical delays, all optical signal regeneration, multicasting and phase sensitive as well as phase insensitive amplification.

Parametric amplification is based on an interaction among four optical frequencies of the electric field propagating through the optical fiber, two of these frequencies may be the same, in a so-called degenerate parametric amplifier. In the interaction, energy is transferred between the interacting waves. The interaction is maximized when the phase-difference between the interacting waves vanish. Consequently, the group velocity dispersion of the used optical fiber is essential. In addition, the interaction depends on the intensity dependent refractive index and consequently highly nonlinear fibers are preferred or fibers with a strong confinement of the electrical field, which produce a high intensity of the electrical field.

Parametric processes and their applications in optical fibers have been subject to research over several decades. However, recent improvements of fiber technology, including highly nonlinear optical fibers and dispersion engineered optical fibers with well controlled dispersion, and availability of high power pump lasers, have seeded new advances in fiber optic parametric amplifiers. In the following focus is directed toward phase insensitive amplifiers and the use in high speed communication systems for regeneration. The essential mechanism that enables regeneration is the saturation performance of the amplifier, which will be described in more detail in this work.

\section{THEORY}

In the following we consider a fiber optical parametric amplifier (FOPA), pumped using a single wavelength pump configuration, where one pump amplifies a signal in a highly nonlinear dispersion shifted fiber. Two photons from the pump at $\omega_{p}$ are annihilated and the power is coupled to the signal at $\omega_{s}$ and to an idler at $\omega_{i}$, as seen in figure $1 \mathrm{a}$.

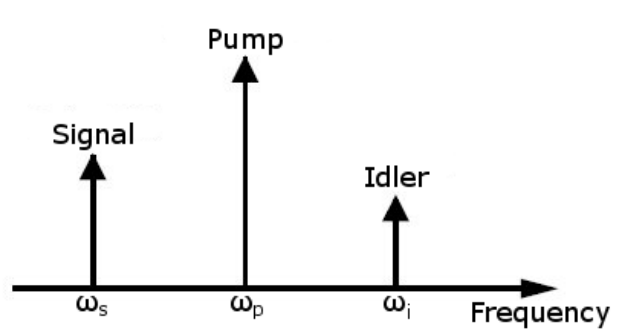

Figure 1a. Illustration of the 3 frequency components of the FOPA: Pump, signal and the generated idler.

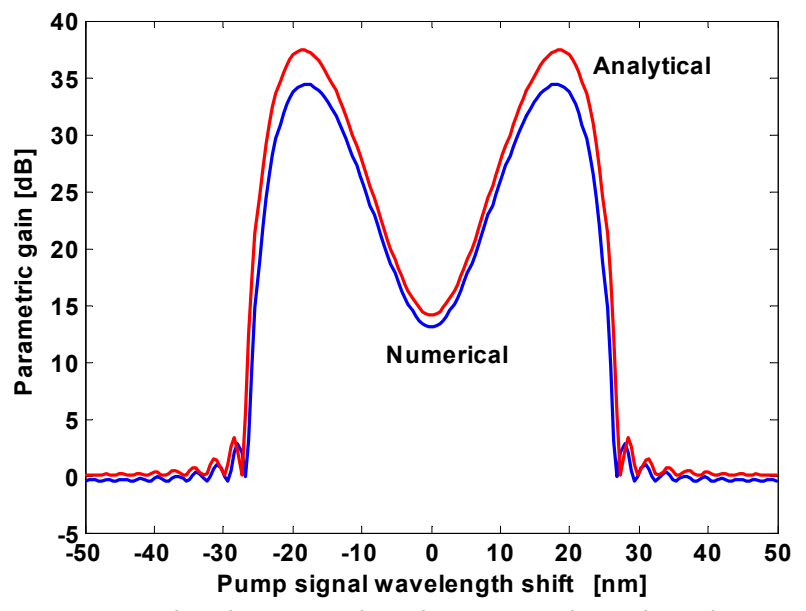

Figure 1b. Theoretical and numerical predicted gain spectrum.

The frequency of the idler is determined by energy conservation: $2 \omega_{p}=\omega_{s}+\omega_{i}$, and the parametric amplification is maximized when the difference between the propagation constants of the three optical waves $\Delta \beta$, matches the nonlinear phase delay per unit length: $2 \gamma P_{p}$. where $\gamma$ is the nonlinear coefficient of the fiber corresponding to its 


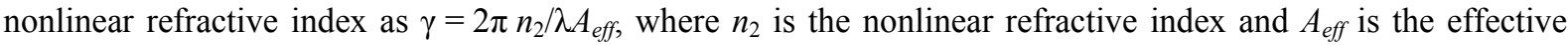
area of the fiber, and $P_{p}$ the power of the pump beam. The difference between the propagation constants are:

$$
\Delta \beta=\beta\left(\omega_{s}\right)+\beta\left(\omega_{i}\right)-2 \beta\left(\omega_{p}\right),
$$

where $\beta\left(\omega_{s, i, p}\right)$ is the propagation constant of the signal, idler and pump, respectively. For this phase match to occur the pump wavelength needs to be close to the zero dispersion wavelength of the nonlinear fiber. In an optical fiber, the envelope of the total electric field $E(x, y, z)$ at a single frequency $\omega$ is written as $E(x, y, z)=U(x, y) A(z)$, where $U(x, y)$ is the transverse mode profile and $A(z)$ is the complex amplitude. The equations that describe the coupling of the amplitudes $A_{p, s, \text { i }}$ of the pump, signal and idler are $[1,2]$

$$
\begin{gathered}
\frac{d A_{p}}{d z}=i \gamma\left[\left(\left|A_{p}\right|^{2}+2\left|A_{s}\right|^{2}+2\left|A_{i}\right|^{2}\right) A_{p}+2 A_{s} A_{i} A_{p}^{*} \exp (i \Delta \beta z)\right]-\frac{\alpha_{p}}{2} A_{p}, \\
\frac{d A_{s}}{d z}=i \gamma\left[\left(\left|A_{s}\right|^{2}+2\left|A_{i}\right|^{2}+2\left|A_{p}\right|^{2}\right) A_{s}+A_{i}^{*} A_{p}^{2} \exp (-i \Delta \beta z)\right]-\frac{\alpha_{s}}{2} A_{s}, \\
\frac{d A_{i}}{d z}=i \gamma\left[\left(\left|A_{i}\right|^{2}+2\left|A_{s}\right|^{2}+2\left|A_{p}\right|^{2}\right) A_{i}+A_{s}^{*} A_{p}^{2} \exp (-i \Delta \beta z)\right]-\frac{\alpha_{i}}{2} A_{i} .
\end{gathered}
$$

In the above, the coupled equations are assumed purely scalar and do not take into account any polarization effects. The amplitudes of the pump, signal and idler are normalized such that the optical power at these frequencies is given by $P_{p, s, i}=\left|A_{p, s, i}\right| 2$. The terms: $-\alpha A_{p, s, i} / 2$ describe the fiber attenuation the pump, signal and idler. In the following it is assumed that the fiber attenuation is independent of wavelength.

When the power of the signal and idler is much smaller that the pump power and when the fiber attenuation is neglected closed form analytical expressions for the gain may be derived [3]. When the power of the signal and/or the idler becomes significant relative to the pump power level or when the loss is not negligible, the coupled equations (2)-(4) needs to be solved numerically using for instance a Runge-Kutta method. For high gain amplifiers semi-analytical approaches have been shown [4].

Fig. 1b shows the calculated gain using the analytical approach and a numerical simulation that is including the fiber loss, and allowing depletion of the pump beam. In this curve as well as in the following all simulations are based on a highly nonlinear fiber with a nonlinear coefficient $\gamma$ of $10(\mathrm{~W} \cdot \mathrm{km})^{-1}$ and a dispersion slope of $0.015 \mathrm{ps} /\left(\mathrm{km} \cdot \mathrm{nm}^{2}\right)$. A zero-group velocity dispersion wavelength of $1559 \mathrm{~nm}$ and a pump wavelength of $1564 \mathrm{~nm}$ have been assumed.

Fig. 1b, shows a typical gain spectrum providing a peak gain of close to $35 \mathrm{~dB}$ and a bandwidth, defined as the wavelength range over which the amplifier provide gain, of $50 \mathrm{~nm}$. It is noted that the spectrum exhibit a maximum gain that occurs for two wavelengths located symmetric around the pump wavelength.

\section{RESULTS AND DISCUSSION}

In the remaining of this work, saturation characteristics of a fiber optical parametric amplifier is discussed, using numerical simulations. Fig. 2a shows the maximum gain as a function of input signal power level for two different fiber lengths: $500 \mathrm{~m}$ and $250 \mathrm{~m}$.

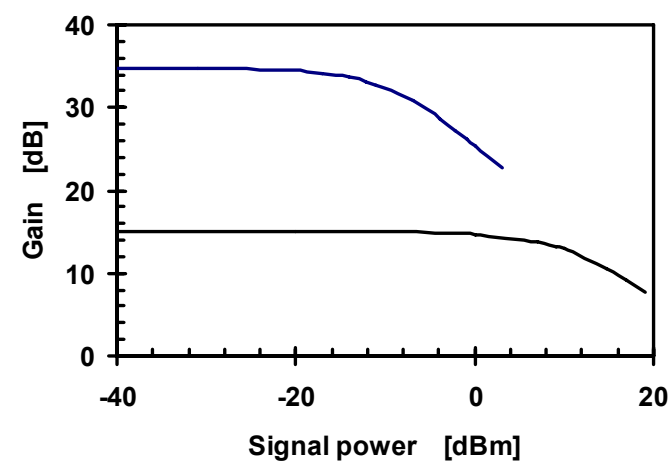

Figure 2a. Gain as a function of input signal power. The upper trace is for a $500 \mathrm{~m}$ long fiber whereas the lower trace is for a $250 \mathrm{~m}$ long fiber.

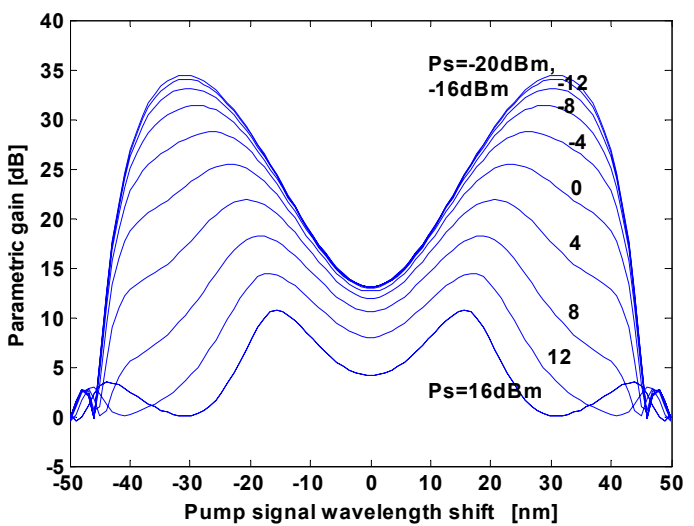

Figure 2b. Gain spectrum for a 500 long fiber using $1 \mathrm{~W}$ of pump power The input signal power is varied from $-20 \mathrm{dBm}$ to $16 \mathrm{dBm}$ in steps of $4 \mathrm{~dB}$ as illustrated on the curves

A fiber attenuation of $0.9 \mathrm{~dB} / \mathrm{km}$ and a fixed pump power level of $1 \mathrm{~W}$ have been used. The figure illustrate that the amplifier is not able to sustain a high as the input signal power increases. Not only the peak gain reduces as 
the signal power increases, also the spectral shape of the gain changes as the input signal power is increased. This is illustrated in Figure 2b. The consequence of the saturation is that the amplifier gain drops, the wavelength where maximum gain appears moves closer to the pump wavelength and finally the bandwidth over which the amplifier provides gain also reduces significantly.

When using the amplifier in a setup for signal regeneration, the amplifier is operated in saturation and consequently the power where the maximum gain has dropped by $3 \mathrm{~dB}$ is an essential parameter. In the following, the power level at which the gain is reduced by $3 \mathrm{~dB}$ is referred to as the saturation power level. It is expected that this saturation power level is a function of the pump power, the fiber length, the attenuation and the nonlinearity. Figure 3 illustrate the saturation power level as a function of pump power for a fixed fiber length of $500 \mathrm{~m}$ for two values of the attenuation $0.9 \mathrm{~dB} / \mathrm{km}$ and $3.6 \mathrm{~dB} / \mathrm{km}$ respectively.

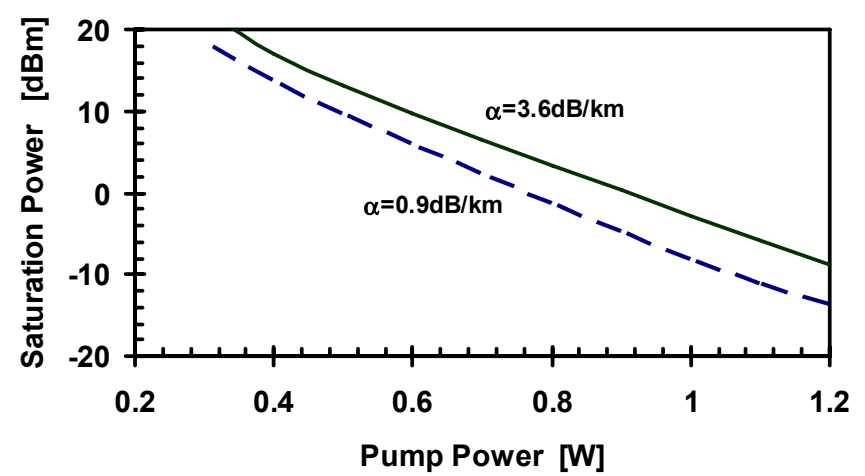

Figure 3. Saturation power level as a function of pump power for a $500 \mathrm{~m}$ long highly nonlinear fiber. Two values of the attenuation is considered: $0.9 \mathrm{~dB} / \mathrm{km}$ and $3.6 \mathrm{~dB} / \mathrm{km}$.

The figure shows that the saturation power scales dramatically with the pump power and less with the fiber attenuation. This is explained by the relatively short length of fiber which is much shorter that the effective length of the amplifier.

To demonstrate reshaping of a high capacity optical signal using a FOPA, we inserted a parametric amplifier into a $40 \mathrm{~Gb} / \mathrm{s}$ system based on return to zero-differential phase shift keyed modulation format. The fiber had a zero dispersion at $1560.5 \mathrm{~nm}$, a dispersion slope of $0.015 \mathrm{ps} /\left(\mathrm{nm}^{2} \cdot \mathrm{km}\right)$, an attenuation of $0.74 \mathrm{~dB}$ and a nonlinear coefficient of $11.5(\mathrm{~W} \cdot \mathrm{km})^{-1}$. The amplifying fiber was $500 \mathrm{~m}$ long and was pumped using a phase modulated pump beam with a power level of $30 \mathrm{dBm}$.. By driving the amplifier into saturation an improvement of close to $4 \mathrm{~dB}$ in the required optical signal to noise ratio for a $10^{-9}$ BER performance was achieved.

\section{CONCLUSION}

Saturation performance of a fiber optic parametric amplifier is shown. Significant changes in the saturation power level and the spectral gain shape is demonstrated as a function of fiber parameters, as for example the fiber length. A system demonstration shows a $4 \mathrm{~dB}$ improvement in the required signal to noise ratio for a fixed bit error rate level.

\section{ACKNOWLEDGEMENTS}

EOARD is thanked for financial support.

\section{REFERENCES}

[1] Hansryd, et al., "Broad-band continuous-wave-pumped fiber optical parametric amplifier with $49 \mathrm{~dB}$ gain and wavelength-conversion efficiency," Photonics Technol. Letters, vol. 13, no. 3, pp. 194, 2001.

[2] T. Torounidis, et al., "Fiber-Optical Parametric Amplifier with 70 dB Gain," Photonics Technol. Letters, vol. 18, no. 10, p. 1194, 2006.

[3] Hansryd, et al., "Fiber-Based Optical Parametric Amplifiers and Their Applications," IEEE J. of Selected Topics in Quantum Electronics, vol. 8, no. 3, pp. 505, 2002.

[4] P. Kylemark, et al., "Semi-Analytical Saturation Theory of Fiber Optical Parametric Amplifiers," J. of Lightwave Technology, vol. 24, no. 8, p. 3471, 2006. 